\title{
La creatividad y los beneficios que causa aplicar en las empresas para la competitividad empresarial
}

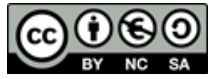

\section{The creativity and benefits it causes to apply in companies for business competitiveness}

Pablo Homero Velasteguí López. ${ }^{1}$

Recibido: 05-09-2018 / Revisado: 17-09-2018 / Aceptado: 21-09-2018 / Publicado: 05-10-2018

Resumen.

DOI: https://doi.org/10.33262/concienciadigital.v1i4.903

La Creatividad no es sólo el proceso de generación de ideas sino también la gestión de las mismas para que se traduzcan en innovaciones que aporten valor a la empresa. Es importante reseñar, la importancia que adquiere la Creatividad a la hora de hacer frente a las situaciones estratégicas, organizativas, competitivas, problemáticas, etc., presentes y futuras que forman parte de la vida empresarial.

Cada autor realiza su propia interpretación acerca de la creatividad, pero desde el punto de vista de esta como origen de la innovación la definiríamos como: "Creatividad supone la creación de algo que es original, que es nuevo y por tanto que se sale de lo habitual y de lo cotidiano pero que además es valioso, es decir, tiene un valor, una utilidad para aquellas personas que lo han generado y para su entorno. El término creatividad se puede entender como el proceso de generación de nuevas ideas o como resultado de ese proceso que acaba con la generación de algo novedoso. En resumen, se entiende por Creatividad los procesos encaminados a la generación de esa idea novedosa y útil porque el resultado entendemos que es la innovación, el cambio que se produce en una organización si dicha idea es puesta en marcha."

Palabras claves: Creatividad, innovaciones, empresa, estratégicas, competitividad.

\footnotetext{
${ }^{1}$ Ciencia digital, Ambato, Ecuador, pablohomerovelastegui@cienciadigital.org
} 


\section{Abstract.}

Creativity is not only the process of generating ideas but also the management of them so that they can be translated into innovations that add value to the company. It is important to note the importance that Creativity acquires when it comes to dealing with the strategic, organizational, competitive, problematic, etc., present and future situations that are part of business life.

Each author makes his own interpretation about creativity, but from the point of view of this as the origin of innovation we would define it as: "Creativity means the creation of something that is original, that is new and therefore that goes beyond what It is a valuable and useful tool for those who have generated it and for its environment, the term creativity can be understood as the process of generating new ideas or as a result of This process ends with the creation of something new, in short, Creativity is understood as the processes aimed at generating this innovative and useful idea because the result we understand is innovation, the change that occurs in an organization if that idea it is set in motion. "

Keywords: Creativity, innovations, company, strategic, competitiveness.

\section{Introducción.}

Según Los Autores (Gardner, 1995; Agüera, 2011; Trigo, 1999). La creatividad implica novedad inicial y aceptación final. - La creatividad se define por la elaboración de nuevos productos o el planteamiento de nuevos problemas. - La creatividad es reconocida como tal sólo cuando ha sido aceptada en una cultura concreta. - Una persona suele ser creativa en un campo y no en todos. - Una persona puede ser llamada creativa cuando exhibe su creatividad en forma consistente.

La creatividad es "tener ideas y comunicarlas". La creatividad por naturaleza es una idea nueva que tiende a hacerse patente a través de la expresión. El proceso creativo culmina con la comunicación porque si no se quedaría tan solo en ideación.

Es decir, la creatividad de una persona radica en la conjunción de una actitud, un conjunto de aptitudes y una manera de trabajar siguiendo un conjunto de reglas, técnicas y métodos. La creatividad de un resultado del proceso de creación (pensamiento, objeto o servicio) radica en la consecución de determinadas características.

\section{Criterio Personal}

Cada uno de los autores da su punto de vista acerca de la creatividad, una persona suele ser creativa en un campo y no en todos puede ser creativa cuando exhibe su creatividad en forma 
consistente ya que reside en la unión de una actitud por la elaboración de nuevos productos o también el planteamiento de nuevos problemas.

La creatividad también consta de objetivos principales de la creatividad en la empresa, basado en la innovación para la mejora de competitividad, los beneficios en la creatividad es un componente que sobresalta a toda la organización empresarial, pues la creatividad es reconocida como tal sólo cuando ha sido aceptada en una ilustración concreta.

La creatividad es pensamiento original, imaginación constructiva, pensamiento creativo, entre otras maneras, es la facultad que alguien tiene para crear y a la capacidad creativa de un individuo. Consiste en encontrar procedimientos o elementos para desarrollar labores de manera distinta a la tradicional, con la intención de satisfacer un determinado propósito.

La creatividad permite cumplir deseos personales o grupales de forma más veloz, sencilla, eficiente o económica. El proceso de creatividad consistirá en encontrar aquellos métodos u objetos más satisfactorios para realizar aquellas tareas que permitan la concreción de maneras o cosas nuevas y distintas, siendo el ingenio la principal fuente de inspiración para dar curso a los mismos.

Además, la creatividad, suele ser una excelente herramienta a la hora de tener que cumplir o satisfacer deseos, ya que nos permitirá hacerlo de manera rápida, efectiva, económica, sin necesidad de embarcarnos en grandes empresas o gestas para lograr aquello que queremos o deseamos.

\section{Objetivo de la creatividad en la empresa.}

Los Autor (Domínguez, 2007; Soto, 2012) sostienen que, El primer y principal objetivo es el desarrollo, tan extenso y preciso como resulte conveniente, de las técnicas necesarias para un adecuado conocimiento y manejo, desde el punto de vista empresarial, además, la realización de los oportunos ejercicios de carácter práctico.

La creatividad en la empresa es un aspecto clave, sobre todo cuando se están creando importantes cambios y se requiere una mayor competitividad. Hay que saber adaptarse, pero mucho más importante es adaptarse con esta ventaja a mayores, de una forma más flexible e innovadora.

\section{Criterio personal}

Es evidente que a lo largo de estas investigaciones trataremos el concepto de creatividad desde un punto de vista empresarial, penetrando para ello en el conjunto de conocimientos que constituyen la llamada trabajo de la creatividad; sin embargo y para fortalecer más este proceso, efectuaremos a lo largo del mismo establecidas precisiones de carácter psicológico y realista que tienen por objeto distintivo el investigar sobre la creatividad empresarial. 
La creatividad, como proceso completo, está compuesta por un conjunto de cambios perfectamente precisados, inmediatos y autónomos entre sí que se elaboran total o en parte según el contorno en el que estemos planteando dicho proceso. Creatividad, en la que definición de un objetivo y mediante la activación de determinados mecanismos de carácter cognitivo, llega a plasmar una idea proporcionada al objetivo buscado.

Los autores nos dicen que los objetivos de la creatividad en la empresa es acortar los periodos de vida de los productos, implantando innovaciones los cuales permitan reemplazarlos por otros extender la acogida mediante la renovación de nuevos productos que den respuesta a la demanda de crecimiento encanto a la calidad de servicios, como abrir sucesos tecnológicos que disminuyan precios y establecer nuevos productos y optimizar el diseño de los productos aumentar los mercados a los que llegan nuestra oferta de productos y servicios y así alcanzar nuevos objetivos de desarrollo tan grandes como resulten convenientes en la creatividad empresarial.

Realización, en la que se proviene a la reconstrucción de la idea obtenida. Tal construcción se llevará a cabo acorde a las técnicas propias del ámbito en el que se esté trabajando. En una segunda forma del proceso algo más complicada pero muy usual, es preciso crear una nueva fase, y es que suele ocurrir que al poner en marcha la acción creativa no se genera una sino varias ideas acordes con el objetivo preestablecido. En tal cuestión y antes de la realización, se hace necesario proceder a una elección, fase en la que se elige aquella idea que, a juicio del promotor o realizadores del proceso, es la más conforme con el propósito que se persigue.

\section{Beneficios de la creatividad aplicada en la empresa.}

Desacuerdo a (Ponti, La Empresa Creativa, 2012; Renart, 2000) El principal beneficio es el aumento de la capacidad competitiva de las empresas y la sustentabilidad a largo plazo. Pero otra gran ventaja es la motivación en las personas: hacerlas participar en el proceso creativo innovador las hace crecer como seres humanos, aumentando la fidelización con las empresas.

Los beneficios que puede obtener la empresa son múltiples. En primer lugar se incrementan los beneficios derivados de mejoras en el valor ofrecido al usuario. De esta manera aumenta el posicionamiento de la empresa y consigue destacar entre las demás, estableciendo un vínculo emocional entre marca-consumidor.

\section{Criterio personal}

Como estamos viendo, la creatividad es un elemento que afecta a toda la organización, la motivación en las personas, hacerlas participes en el proceso creativo hace que las personas crezcan como seres humanos, pasando por el centro de decisión y llegando a todas las áreas y departamentos de la empresa, de esta manera se consiguen destacarse entre las demás. 
La utilización de enfoques y técnicas creativas permite alimentar y mejorar los procesos de innovación, que es el elemento diferencial de las empresas que se posicionan en la vanguardia del mercado ya que se incrementa los bienes provenidos de mejoras en el coste brindado al usuario y así aumenta la mejora de la empresa.

También nos dice que los beneficios de la actividad creativa en la empresa se pueden clasificar en cuatro grandes grupos.

1. Desarrollo del negocio.

2. Relación con el cliente.

3. Nuevas oportunidades.

4. Mejora de la competitividad.

La creatividad ayuda a generar diferentes modelos de negocio, implementando técnicas de trabajo más participativas, aumentando aportes de los trabajadores, como cualidades personales, tiempo para imaginar, confianza y disposición entre otras más, que ayudan a los beneficios de la creatividad.

\section{Características de la persona creativa.}

Según los Autor (Ramirez, 1991; Ponti \& Ferras, 2008) Los autores dicen que las características de las personas creativas son las siguientes:

Curiosidad.- la cualidad de cuestionar y trata de saber más sobre el mundo que lo rodea.

Imaginación.- la persona rebasa las fronteras de lo real o convencional en el proceso generativo de ideas.

Mente abierta.- tiene la capacidad de aceptar que otras personas también pueden tener buenas ideas y la actitud de disponibilidad para escucharlas.

Capacidad de concentración.- tenacidad para continuar con un punto de interés independiente de ambiente que lo rodea.

Persistencia.- capacidad para continuar una tarea hasta terminarla o encontrar suficientes razones que la obligue abandonarla.

Inteligencia.- es importante aunque en estudios realizados se ha encontrado que esta no es una cualidad fundamental para la creatividad.

Independencia.- le agrada trabajar por su lado, siguiendo sus propias ideas e inclinaciones.

Responsabilidad.- La persona es consciente de los compromisos personales y sociales que contrae. 
Imprecisión.- no se ajusta a las reglas, salta en ideas de una a otra es generalista con un criterio amplio. Otras cualidades de carácter personal se le atribuye a la persona creativa como el ser distinta sin tratar siquiera de serlo, enérgica, compulsiva, impredecible, no convencional, escéptica, sensitiva y aventurera.

Búsqueda constante de ideas.- No son creativas en un momento particular y luego dejan de serlo, sino que convierten esa característica en un hábito y un una forma de serlo. Su mente es abierta en ebullición, generan ideas para cualquier reto que se les plantea y abren caminos y vías de solución para los problemas que surgen alrededor.

Actitud transgresora.- Una persona creativa es, por definición, inconformista. No le gustan que las cosas siempre estén igual, aunque estén bien, en cualquier caso pugna a mejorarlas.

\section{Criterio personal}

Las características de las personas creativas exceden las fronteras de lo real o convenido en el proceso generativo de ideas, sin que sea una obligación que se deban poseer manifiestan una actitud abierta frente a su entorno, es decir se mueven, piensan y hacen cosas constantemente ya que son muy críticas.

También se adaptan a sus peculiaridades condiciones son capaces de liberarse de las supuestas intuiciones impuestas por la tradición, tienen mucha fluidez y por eso elevan su capacidad para generar ideas, buscan el éxito independientemente de lo que digan los demás, aman la libertad, son muy inconformistas ya que no les gusta todas las cosas estén igual siempre buscan mejorarlas.

Es decir son aquellas que son capaces de manifestar nuevas formas de orientar un problema o de plantear solución a estos utilizando mecanismos lógicos no convencionales. Se distinguen por su habilidad para adaptarse a todo tipo de situaciones y por ser capaces de alcanzar los objetivos que se plantean.

Para lograrlo, estos individuos recurren a mecanismos complejos y es precisamente estos modos, la complejidad, la que les diferencia en mayor medida del resto de personas.

\section{Características de la organización creativa.}

Para los autores (Infinitas, 2016; Xavier Greffe, 2006). Es una distribución de personas que trabajan para uno o más objetivos o metas comunes, entre los cuales se respira un ambiente propicio para el pensamiento creativo.

* El éxito de un equipo es motivo de celebración para todos.

* Inspiran a todos los niveles de organización.

* Fomentan crítica constructiva. 
Tiene la transparencia por bandera.

* Priorizan a las personas en tiempos difíciles.

* Explican la misión de trabajo.

* Cuidan las relaciones emocionales.

Una situación importante es considerar que desarrollar la creatividad no es sólo emplear técnicas atractivas o ingeniosas por sí mismas; desarrollar la creatividad implica incidir sobre varios aspectos del pensamiento; las cuatro características más importantes del pensamiento creativo son:

* La fluidez

* La flexibilidad

* La originalidad

* La elaboración

\section{Criterio personal}

Los autores nos dicen que cada triunfo obtenido es celebrado por todos y eso inspira a las demás personas a seguir adelante, dan críticas constructivas para que cada uno mejore con el transcurso del tiempo cada quien explica el porqué de su trabajo, la creatividad no solo es utilizar técnicas atrayentes o ingeniosas por sí mismo, la fluidez es la capacidad de generar ideas y la flexibilidad nos ayuda a mejorar nuestras alternativas en diferentes ámbitos, la originalidad consiste en argumentar ideas que jamás a nadie se le ha ocurrido anteriormente, la elaboración también es muy relevante ya que mediante esto ha ido desarrollándose la empresa.

Para ampliar la creatividad implica incurrir sobre diversos aspectos del pensamiento:

La fluidez: se describe a la capacidad de crear una cantidad enorme de ideas o respuestas a ideas formadas.

La flexibilidad: Manejar nuestras opciones en diferentes campos o categorías de respuesta, es voltear la cabeza para otro lado buscando un enfoque más amplio, o diferente a la que siempre se ha visto

La originalidad: Es el semblante más propio de la creatividad y que envuelve pensar en ideas que nunca a nadie se le han ocurrido o imaginar los problemas de otro modo.

La elaboración: Una particularidad significativa en el pensamiento creativo es la producción, ya que a partir de su manejo es como ha ido evolucionado más las empresas. 


\section{Gestión científica versus gestión artística en la empresa}

(Barreiro, Castro, Fernandez, Santamarin, \& Perez, 2003; John M, 1996; Idea B., s.f.). Nos dicen que existen dos tendencias para gestionar la empresa.

\section{Gestión científica.}

Es aquella forma de saber consistentemente en acumular conocimientos mediante la utilización de técnicas o métodos adecuados.

Transferencia de tecnología. La transferencia de la tecnología puede tener lugar desde una unidad de una compañía a otra unidad de esta. La transferencia de tecnología se define como el proceso de aplicación de conocimientos.

Gestión artística. Esta concepción deja a merced de la improvisación y de la creatividad de los trabajadores de la empresa el destino de la empresa. Al contrario que en el caso anterior, esta visión de la empresa se caracteriza por la exploración continua, es un hervidero de ideas altamente creativas y potencialmente valiosas, pero la falta de una estrategia para su explotación le impide obtener recursos, lo que inexorablemente desemboca en el fin del negocio.

\section{Criterio personal}

Existen dos tendencias contrapuestas en la forma de gestionar la empresa: una primera que aboga por la gestión entendida como una ciencia, basadas en la utilización de herramientas tecnológicas y estadísticas, y otra en la que la capacidad de competir de la empresa recae enteramente en la capacidad de improvisación y artística de la empresa.

Las empresas son cada vez más grandes y complejas y requieren de nuevas herramientas para su gestión. Este tipo de herramientas que incorporan mejoras tecnológicas y de control estadístico son las únicas capaces de asegurar que la gestión de grandes empresas transnacionales sea factible y viable.

Por las condiciones del entorno, que obliga a considerar un mayor número de variables, la toma de decisiones en las organizaciones basada en el instinto ya no resulta apropiada y cada vez es más necesaria la utilización de técnicas y software que incorporen algoritmos. Sin embargo, la sistematización de todos los procesos no da pie a la exploración, y se concentra en la explotación de los resultados existentes.

Esta concepción deja a merced de la improvisación y de la creatividad de los trabajadores de la empresa el destino de la empresa. Al contrario que en el caso anterior, esta visión de la empresa se caracteriza por la exploración continua, es un hervidero de ideas altamente creativas y potencialmente valiosas, pero la falta de una estrategia para su explotación le impide obtener recursos, lo que inexorablemente desemboca en el fin del negocio. Ambas 
formas de gestión no solamente son completamente opuestas sino que asimismo muestran formas extremas de entender la gestión empresarial que, sin embargo, llegan a darse en el entorno empresarial. La conclusión, por tanto, es que una gestión eficiente.

Además de estar adaptada a las necesidades de cada caso, resulta de la búsqueda de un equilibrio entre ambas concepciones. Se necesita de una cierta cantidad de exploración para ser creativos (caos creativo), pero esta cantidad de ninguna manera puede constituir el principal activo de la organización.

\section{Factores de la innovación en la empresa.}

Para (John M, 1996; Innovación, 2016). Innovación es la generación de una nueva idea y puesta en práctica en un nuevo producto, proceso o servicio, que es conducente para el crecimiento económico de la empresa, el aumento de empleo y la generación de beneficios.

La Unión Europea define la Innovación en su sentido amplio como la introducción de un nuevo, o significativamente mejorado, producto (bien o servicio), de un proceso, de un nuevo método de comercialización o de un nuevo método organizativo, en las prácticas internas de la empresa, la organización del lugar de trabajo o las relaciones exteriores.

Factores que fortalecen el desarrollar los procesos de innovación en la empresa.

- Análisis de las tendencias de la economía, hábitos de vida y comportamientos sociales.

- Salir de nuestro ámbito de negocio (observar otros mercados geográficos, otros sectores, etc.).

- Aprender de las experiencias de otros, profundizar en sus éxitos y fracasos.

- Conocer los centros de investigación relacionados con nuestro sector y los últimos avances.

- Análisis de las necesidades de nuestros clientes y un feedback más directo con ellos.

- Rotación de puestos de trabajo, formación interna que provoque actitudes favorables al cambio.

- Dotar de recursos y generar equipos horizontales de proyectos.

- Propiciar la creatividad y la participación aceptando el error.

\section{Criterio personal}

Generar nuevas ideas y ponerlas en práctica en nuevos productos o servicios ya que ayuda al crecimiento económico de la empresa gracias a esto podemos generar empleo y beneficios, es considerado un nuevo método de comercialización y organizativo de la empresa. 
También tenemos 7 factores que fortalecen el desarrollo en la empresa estos nos ayudan a analizar hábitos de vida, observar nuevos mercados, conocer nuevos sectores para seguir avanzando, analizar cuáles son las necesidades de nuestros clientes, tener una formación interna de rotación de trabajos, pero también se pueden aceptar errores.

Creatividad, es el proceso en el que, mediante la diligencia de técnicas ajustadas, se inventa siempre un paquete de sucesos y opiniones dirigidas a solucionar un problema existente. En establecidas ocasiones, la actividad creativa puede manipular también, en una fase antepuesta, para la detección de problemas existentes, pero aún no descubiertos, se procede a elegir aquella o aquellas ideas que cumplen en forma ordenada con la condición de utilidad.

Llaman a esta etapa innovación, lo que desde un punto de vista conceptual resulta lógico; sin embargo, en el campo empresarial que es el que agrupa nuestra exposición, la palabra innovación, relacionada a la de gestión, se maneja habitualmente para elegir el proceso completo, por lo que hemos resuelto, en la finalidad de evitar desórdenes, utilizar como nueva denominación de esta fase la de selección.

\section{Los primeros pasos para la innovación en la empresa.}

Según (Cordero, 2013; Navarra, 2008). La innovación es un proceso formal que cuenta con una metodología y herramientas propias que permiten, de una manera estructurada y sistematizada, crear innovaciones.

* Crear dentro de su plan de negocio la estrategia de innovación.

* Desarrollar los nuevos productos o servicios.

* Vigilancia tecnológica.

* Gestión de proyectos tecnológicos y de innovación.

* Financiamiento de la innovación.

* Aseguramiento de la innovación.

* La explotación de la innovación en la estrategia del negocio.

* Conservación y uso del conocimiento de la empresa.

\section{Todo proceso parte, inicialmente deben tener un plan estratégico:}

* Definir de los objetivos de empresa.

* Analizar las fortalezas competitivas.

* Generación de nuevas ideas.

* Tener carácter creativo. 
Las ideas aportadas no tienen por qué ser técnicas, si bien deben suponer actuaciones de tipo innovador que aportan aspectos diferenciales respecto a la competencia y/o constituyen una novedad, al menos para el sector donde compite actualmente la empresa.

\section{Criterio personal}

Para fomentar la Innovación en la empresa nos dice que puedes fortalecer una serie de factores con los que desarrollar los procesos de innovación, deben tener habilidad de realizar innovaciones, ampliar los nuevos productos o servicios tener una visión tecnológica muy clara, contar con creación de estrategias sobre todo mantener sus ideas dentro de la empresa ya que todo negocio inicia de un plan importante, puntualizar sus ideas y así alcanzar los objetivos propuestos para el bienestar de la empresa la Investigación, es factor muy importante en él se realizan uno de conjunto de trabajos teóricos o experimentales, realizados por lo frecuente en departamentos o centros dominados, que se hacen con el objeto de lograr nuevos conocimientos, sin que necesariamente hayan de llevar de inmediato a metas de tipo práctico. Esta fase suele funcionar con libertad de las restantes del proceso y cuando éste se pone en marcha por alguna necesidad concreta, acude en su momento a la investigación pudiendo ocurrir que ésta tuviera ya resueltos los problemas que dicho proceso demanda.

\section{La competitividad empresarial}

Los Autores (Ruiz Gonzàles \& Mandado Pèrez, 1989; Treviño, 2002) sostienen que. Un análisis y seguimiento de los competidores para detectar sus puntos fuertes y débiles, así como sus éxitos y fracasos, proporciona un valiosísimo material informativo sobre posibles fuentes de innovación. Nada tan interesante como apreciar en el competidor la mejor oportunidad de progresar uno mismo.

La competitividad de las empresas es de la mayor urgencia que estas incrementen su esfuerzo tecnológico y de innovación para revertir los efectos de la apertura y la globalización, elevar la competitividad a fin de generar empleos mejor remunerados y crear empresas de bases tecnológicas.

\section{Criterio personal}

La superioridad competitiva es tanto su tejido industrial y económico, depende de la capacidad para renovar, la única ventaja competitiva sostenible es la innovación permanente.

Se define como la capacidad de la empresa para suministrar productos prestar servicios con la calidad deseada y exigida por sus clientes al costo más bajo posible hace referencia a las diferentes estrategias y métodos que las diversas entidades comerciales llevan a cabo con tal de no sólo obtener los mejores resultados. 
Es preciso recalcar que este beneficio concluido puede hacer reseña a cualquier plan dentro de la actividad empresarial, desde la reforma de un proceso administrativo o de elaboración hasta el lanzamiento de un nuevo producto comercial, pasando por la construcción o adquisición de unos nuevos procesos. La ejecución del proceso creador dentro de la empresa se requiere, forzosa e inevitablemente, el adecuado desarrollo de las fases que señalamos y con el grado de tecnificación que para cada una se ha mencionado, por otra parte muy frecuentes, están llamadas a originar soluciones incompletas, en muchos casos con apariencia correcta, pero que suponen una merma importante de imposible reparación, en el resultado final de los planes elaborados.

\section{Datos estadísticos}

De acuerdos a (OMPI, la Universidad Cornell, el INSEAD y los socios especializados del Índice Mundial de Innovación de 2017, la Confederation of Indian Industry, PwC's Strategy \& y la Confederación Nacional de Industria (CNI) y el Servicio brasileño de apoyo a las micro y pequeñas empresas (Sebrae) junio 2017)

Suiza, Suecia, los Países Bajos, los Estados Unidos de América (EE.UU.) y el Reino Unido son los países más innovadores del mundo, mientras que un grupo de naciones, incluida la India, Kenya y Viet Nam aventajan a otras naciones que tienen el mismo grado de desarrollo.

Las principales conclusiones apuntan a que la India está pasando a ser un nuevo polo de innovación en Asia, a los buenos resultados en materia de innovación para el desarrollo en África Subsahariana, y a que existen oportunidades de mejorar la capacidad de innovación en América Latina y el Caribe.

Cada año, el Índice Mundial evalúa la situación en 130 economías mediante docenas de parámetros, desde la presentación de solicitudes de patente, al gasto en educación, y ofrece así un panorama muy completo a los encargados de la toma de decisiones, acerca de la actividad innovadora que es cada vez más un motor de crecimiento económico y social. Este año, el Índice Mundial cuenta con una sección especial en la que se indican los focos principales de invención, es decir, dónde se concentra la mayor densidad de inventores que constan en solicitudes internacionales de patente.

En el Índice Mundial de Innovación, del que este año se publica su décima edición, se señala que sigue habiendo un desfase en la capacidad innovadora entre países desarrollados y países en desarrollo y se observa que es mediocre el índice de progresión en actividades de investigación y desarrollo, tanto a nivel estatal como de las empresas. 


\section{Datos estadísticos}

Figura N 1. Importaciones de bienes por tipo de producto

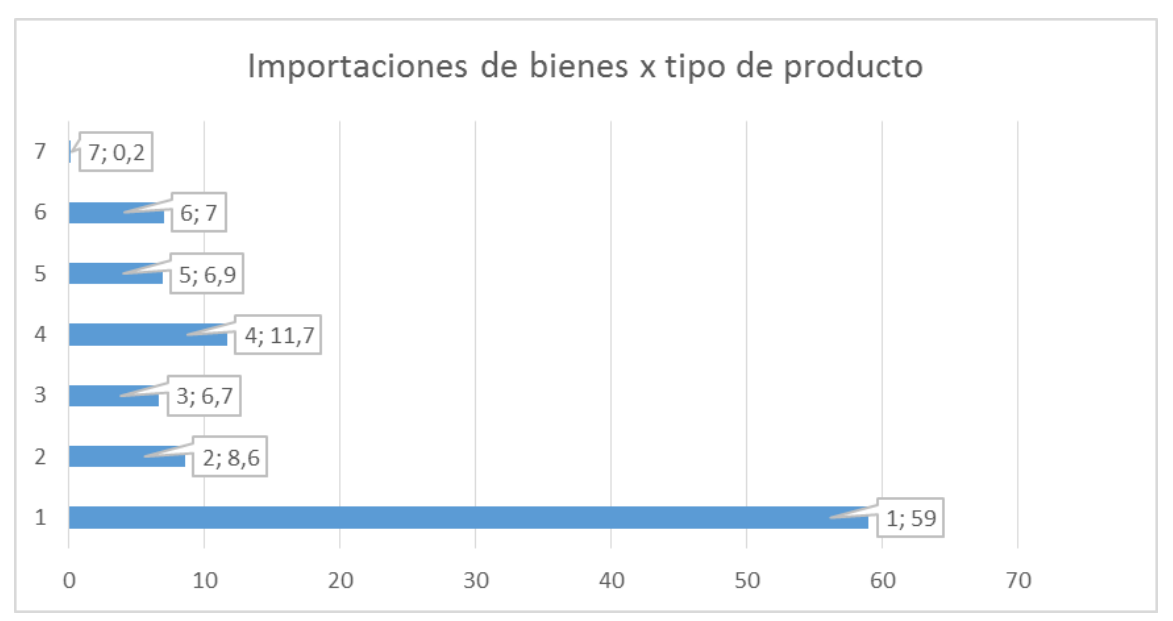

Fuente: Informe de economía creativa 2010

En el siguiente cuadro observamos a que hace referencia cada ítem

Tabla N 1. Tipo de bien o servicio

\begin{tabular}{|l|c|}
\hline 1 & Diseño \\
\hline 2 & Nuevos Medios \\
\hline 3 & Artes Escénicas \\
\hline 4 & Publicaciones \\
\hline 5 & Artes Visuales \\
\hline 6 & Artesanías \\
\hline 7 & Audiovisuales \\
\hline
\end{tabular}

\section{Criterio Personal}

Esto se refiere al tipo de materia prima traída de otro país para ser utilizada en proyectos empresariales, donde se ve claramente que los productos de diseño encabezan la lista y en último lugar están los productos audiovisuales. 
Figura N 2. Índice mundial de innovación en América

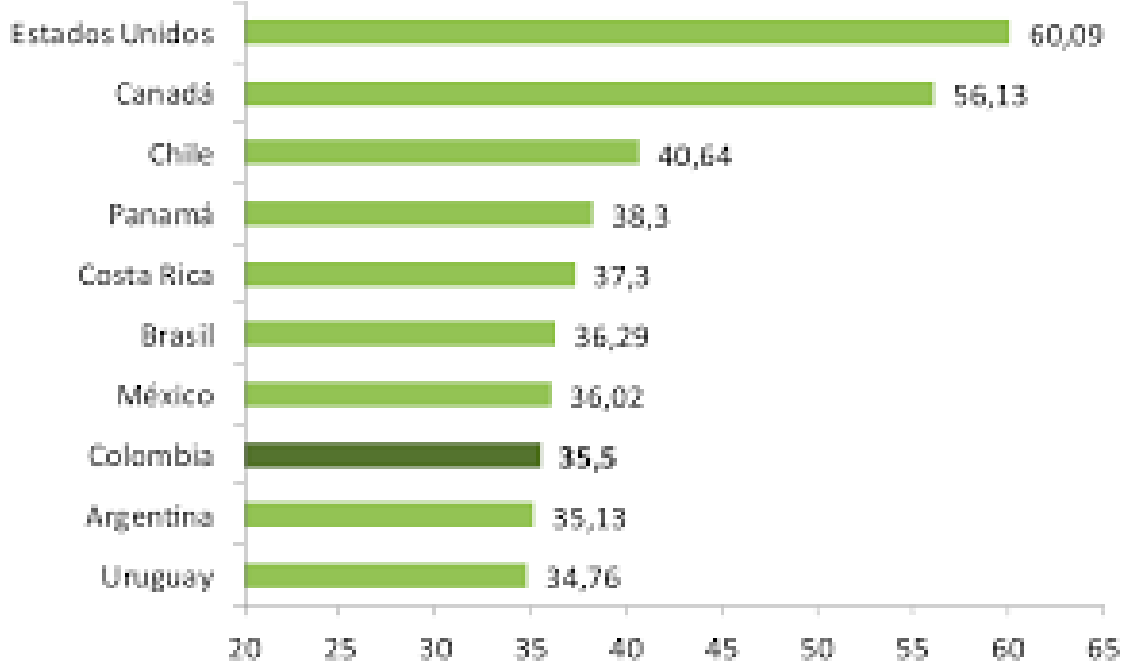

Fuente: Observatorio de desarrollo económico

\section{Criterio Personal}

En América el índice mundial de innovación lo encabeza estados unidos y Canadá mientras que por Sur América tenemos a Brasil y Centro América tenemos a Panamá.

Figura N 3. Actividades profesionales y científicas en el ecuador por provincia

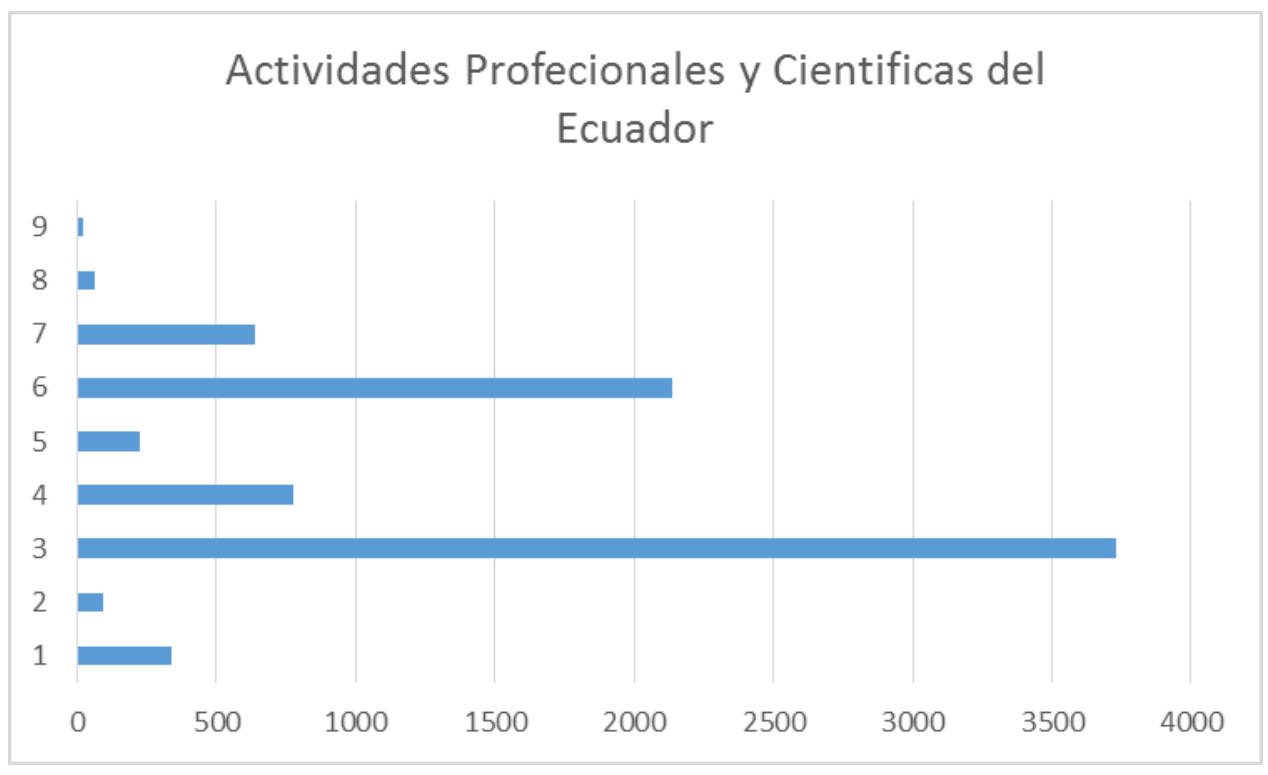

Fuente: INEC

En el siguiente cuadro observamos el significado de los ítems. 
Tabla N 2. Provincias consultadas para el estudio

\section{Criterio personal}

\begin{tabular}{|l|c|}
\hline 1 & Cotopaxi \\
\hline 2 & Bolívar \\
\hline 3 & Pichincha \\
\hline 4 & Tungurahua \\
\hline 5 & Esmeraldas \\
\hline 6 & Guayas \\
\hline 7 & Manabí \\
\hline 8 & Orellana \\
\hline 9 & Galápagos \\
\hline
\end{tabular}

Estos datos fueron sacados directamente de la página del INEC ya que las actividades científicas y profesionales van directamente relacionadas con los proyectos de innovación para lo cual vemos que la provincia de Pichincha encabeza la lista de desarrollo de esta actividad mientras la provincia de galápagos es la que menos realiza esta actividad también se observa a esmeraldas y Cotopaxi que haciendo una comparación respecto al número de habitantes Cotopaxi posee una mayor cantidad de desarrollo en esta actividad lo que justificaría la crisis que hoy en día está pasando esta provincia de la Costa ecuatoriana.

\section{Conclusiones}

- La creatividad empresarial es un elemento que a ha venido interactuando en el entorno empresarial, pero que a medida que los mercados se hacen más competitivos, ya que por medio de estos las organizaciones pueden desarrollar ventajas competitivas que les permiten mantenerse con éxito.

- La creatividad e innovación empresarial se torna necesaria independientemente de la índole o el giro de la empresa de la que estemos hablando ya que cada vez existe más competencia en el ámbito laboral.

- Hoy en día en nuestra existe una competencia global, en la cual no debemos ser conformistas, por el contrario, tenemos que seguir creando ideas nuevas.

- La idea final a la que nos conducen los distintos aspectos analizados en este estudio es la de considerar que para el afloramiento de la creatividad en las organizaciones empresariales se precisa actuar de forma simultánea en varios sentidos. 


\section{Referencias bibliográficas.}

"Idea", ". B. (s.f.). Manual de la Creatividad Empresarial.

"Idea", ". B. (s.f.). Manual de la Creatividad Empresarial.

"Idea", C. B. (s.f.). Manual de la Creatividad Empresarial.

Agüera, L. G. (2011). Anatomía de la Creatividad. ESDi - Unitat Departamental de Teoria i Anàlisi del Disseny.

Barreiro, Castro, D. d., Fernandez, B., Santamarin, R., \& Perez, L. (2003). Gestión Cientifica Empresarial. Coruña: Netbiblo SI .

Ceei Alcoy: Factores que favorecen la innovación en la empresa. (14 de Abril de 2016). Obtenido de http://ceeialcoi.emprenemjunts.es/?op=8\&n=12253

Cordero, R. (2013). Innovación, Negocios, Pymes.

Domínguez, R. A. (2007). La creatividad en la empresa. Alianza Editorial.

Gardner, H. (1995). Mentes Creativas.Una Anatomía de la Creatividad Humana. Barcelona: Paidós.

González, J. M. (2002). La Ciencia y la Empresa. Monterrey.

Idea, C. B. (s.f.). Manual de Creatividad Empresarial. 21-22.

Imagen Profesional Line. (s.f.). Obtenido de http://www.facpce.org.ar:8080/iponline/elsistema-de-control-interno-y-su-importancia-en-la-auditoria/

infinitas, I. (2016). Características de las organizaciones creativas.

Innovación, C. E. (2016). Ceel Alcoy. Obtenido de http://ceeialcoi.emprenemjunts.es/?op $=8 \& n=12253$

John M, I. (1996). Gestiòn Calidad y Competitividad.

Luis, G. d. (2006). Creatividad. Madrid.

Mayer, R. E. (1983). Pensamiento, resolución de problemas y cognición. Paidos Iberica.

Mintzberg. (1991). Mintzberg y la dirección. Madrid: Diaz Santos S.A. 
Navarra, A. d. (2008). La gestión de la Innovación.

Ponti, F. (2012). La Empresa Creativa. Granica.

Ponti, F., \& Ferras, X. (2008). Pasión por innovar un modelo novedoso que incentiva la creatividad empresarial. Grupo Editorial Norma.

Ramirez, E. M. (1991). Calidad Integral Empresarial e institucional (Primera ed.). Grupo Noriega.

Renart, J. B. (2000). Creatividad Aplicada a la Empresa. Barcelona: Ediciones Gestiòn.

Ruiz Gonzàles, M., \& Mandado Pèrez, E. (1989). La innovación tecnológica y su gestión. Barcelona.

Soto, B. (2012). Gestión Org. Obtenido de https://www.gestion.org/recursoshumanos/31640/la-importancia-de-la-creatividad-en-las-empresas/

Taraquez, Edwin; Lima, Carolina. (2013). Creatividad Empresarial (Segunda ed.). Bogota: Ecoe Ediciones.

Treviño, L. C. (2002). Innovación y competitividad empresarial. Puebla, Mexico.

Trigo, E. (1999). Creatividad y Motricidad. Barcelona: Inde.

Velasco, J. P. (2008). Gestión de la claidad Empresarial. Madrid: Esic.

Villaverde, L. M. (2006). Gestión del cambio y la innovación en la empresa. (Vol. I). Vigo.

Xavier Greffe, c. I. (2006). Gestión de empresas.

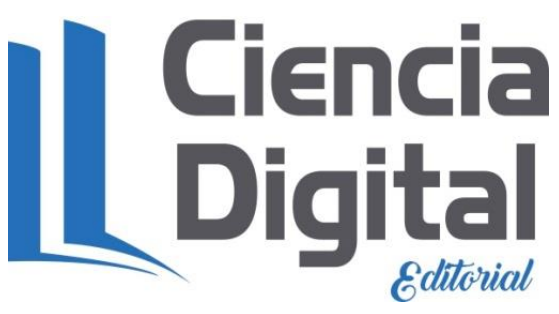


Para citar el artículo indexado.

Velasteguí López, P. (2018). La creatividad y los beneficios que causa aplicar en las empresas para la competitividad empresarial. ConcienciaDigital, 1(4), 6-23. https://doi.org/10.33262/concienciadigital.v1i4.903

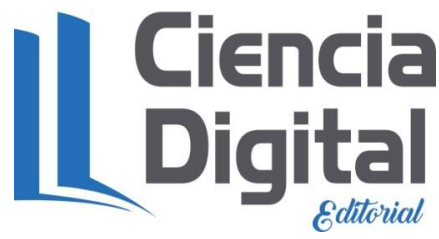

El artículo que se publica es de exclusiva responsabilidad de los autores y no necesariamente reflejan el pensamiento de la Revista Conciencia Digital.

El articulo queda en propiedad de la revista y, por tanto, su publicación parcial y/o total en otro medio tiene que ser autorizado por el director de la Revista Conciencia Digital.
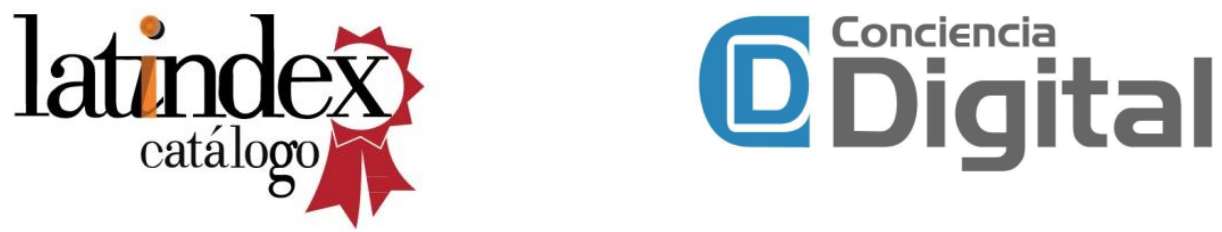